\title{
Weight Control Methods Related to Cotinine-Verified Smoking among Korean Adult Women: Korea National Health and Nutrition Examination Survey, 2008-2011
} Article

Young Kyun Kim, Young Gyu Cho*, Jae Heon Kang, Hyun Ah Park, Kyoung Woo Kim, Yang Im Hur, Yeon Gak Yoo, Jiyoung $\mathrm{An}^{1}$

Department of Family Medicine, Inje University Seoul Paik Hospital, Inje University College of Medicine; u-Healthcare Design Institute, Inje University, Seoul, Korea

\section{ABSTRACT}

Background: Korean women are known to have a very low smoking rate. However, the actual smoking rate among Korean women is higher than $10 \%$ and may continue to increase gradually. In addition, some Korean women use extreme weight control methods that have potentially harmful effects. This study was conducted to elucidate weight control methods related to cotinine-verified smoking among Korean adult women.

Methods: This cross-sectional study involved 4,189 women aged $\geq 19$ years who had attempted weight control during the past 1 year from the Korea National Health and Nutrition Examination Survey, 2008-2011. Smoking status was assessed using both self-report questionnaires and assays of urinary cotinine, and weight control methods were investigated using self-report questionnaires.

Results: The smoking rate based on the measurement of urinary cotinine was $12.4 \% \pm 0.8 \%$ among Korean women. Cotinine-verified smokers were more likely to attempt fasting (odds ratio, 2.19; 95\% confidence intervals, 1.03 to 4.67), taking prescription diet-pills (odds ratio, 2.37; 95\% confidence intervals, 1.47 to 3.82), and taking nonprescription diet-pills (odds ratio, 3.46; 95\% confidence intervals, 1.71 to 6.98), and were less likely to attempt eating less food or modifying dietary patterns (odds ratio, $0.71 ; 95 \%$ confidence intervals, 0.51 to 0.99 ) compared to non-smokers.

Conclusion: Korean adult women's smoking is independently related to a high likelihood of using weight control methods with potentially harmful effects, such as fasting and taking diet-pills, and a low likelihood of choosing weight control methods, including dietary modification, that require constant effort for a prolonged time.

Keywords: Smoking; Cotinine; Body Weight; Weight Loss; Korea; Women

Received: August 26, 2014, Accepted: February 26, 2015

*Corresponding Author: Young Gyu Cho

Tel: +82-2-2270-0097, Fax: +82-2-2267-2030, E-mail: jacobel@hanmail.net

Korean Journal of Family Medicine

Copyright (C) 2015 The Korean Academy of Family Medicine

This is an open-access article distributed under the terms of the Creative Commons Attribution Non-Commercial License (http://creativecommons.org/licenses/by-nc/3.0) which permits unrestricted noncommercial use, distribution, and reproduction in any medium, provided the original work is properly cited. 


\section{INTRODUCTION}

The smoking rate among Korean women is reported to be very low $(<10 \%)$, which is appreciably less than that of Western women. ${ }^{1)}$ However, several recent studies have reported the smoking rate to exceed $10 \%$ among Korean women when using biochemical markers, such as urinary cotinine. ${ }^{2,3)}$ This dichotomy suggests that surveys that depended on self-report could have underestimated the smoking rate among Korean women. This under-reporting may reflect false reporting by Korean women smokers under societal pressure against women's smoking in Korean society.

There is a gender difference in smoking motives. The use of smoking as a means of weight control is approximately double in women than in men. ${ }^{4)}$ The relationship between women's smoking and weight control has been studied mostly with adolescents and young adult women. Weight control was positively related to current smoking in female adolescents. ${ }^{5)}$ Both dieting and smoking may be included in the constellation of adolescent problem behaviors. Adolescents who use extreme weight control methods, such as vomiting and diet-pills, can be at increased risk for engaging in healthcompromising behaviors, such as tobacco, alcohol, and marijuana use. ${ }^{6}$

Wee et al. ${ }^{7)}$ reported that US adults younger than 30 years were more likely to smoke if they were trying to lose weight, whereas in Americans aged $\geq 30$ years, weight control efforts bore no relationship to smoking status. Since many older adults seek to control their weight for improved health or disease management, the relationship between smoking and weight control among older adults may be different from that of adolescents and young adults. However, Korean women smokers may have different characteristics from Western women smokers because the societal pressure against women's smoking in Korea differs from that in Western societies. Lee et al. ${ }^{3)}$ demonstrated that Korean women smokers who engaged in weight control attempts were more likely to lie about their smoking status. Many Korean women strive for weight control regardless of their weight status due to a socio-cultural preference for thinner women in Korean society. ${ }^{8}$ Yet, no study has addressed the relationship between women's smoking and weight control methods, such as exercise, fasting, and taking diet-pills, among Korean adult women.

This study was conducted to elucidate weight control methods related to cotinine-verified smoking among Korean adult women who had attempted weight control during the past 1 year.

\section{METHODS}

\section{Study Subjects}

This study was based on data from the Korea National Health and Nutrition Examination Survey (KNHANES), 2008-2011. KNHANES is a nationwide representative survey using a complex, stratified, multistage, cluster-sampling design that examines the general health and nutritional status 
of the entire Korean population. The survey has been conducted annually since 2007 using a rolling sample design. ${ }^{9)}$ A total of 7,450 women aged $\geq 19$ years who participated in the KNHANES, 20082011, were assessed for their smoking status using both self-report questionnaires and assays of urinary cotinine. Among them, 4,251 women responded that they had, on their own initiative, tried to lose or maintain their body weight during the past 1 year. Finally, we analyzed the data of 4,189 women after excluding women whose height or weight data was missing $(\mathrm{n}=11)$, pregnant women $(\mathrm{n}=35)$, and women with a history of renal failure or serum creatinine $\geq 1.5 \mathrm{mg} / \mathrm{dL}(\mathrm{n}=16)$.

\section{Measurements}

1) Smoking status

Smoking status was assessed using the subjective responses given in the self-report questionnaires as well as using an objective measurement of urinary cotinine. Urinary cotinine level was measured using the Tandem mass API 4000 (Applied Biosystems, Carlsbad, CA, USA) or Perkin Elmer Clarus 600T (PerkinElmer, Turku, Finland). Subjects with urinary cotinine levels $\geq 50 \mathrm{ng} / \mathrm{mL}$ were defined as cotinine-verified smokers. ${ }^{10)}$ Smoking status based on self-report was classified into three categories. Subjects who reported smoking at least 100 cigarettes during their lifetime and reported smoking at the time of the survey were defined as current smokers (self-reported smokers). Subjects who reported smoking at least 100 cigarettes during their lifetime but reported not smoking currently were defined as ex-smokers. Subjects who reported smoking fewer than 100 cigarettes during their lifetime were defined as non-smokers.

\section{2) Weight control methods}

Subjects who had tried to lose or maintain their body weight during the past 1 year were asked to report every method that they had used to control their weight during that time. The queried weight control methods included exercise, eating less food or modifying dietary patterns, one-food diet, fasting, taking dietary supplements, taking Korean herbal medicine, taking prescription diet-pills, and taking nonprescription diet-pills. The subjects were allowed to choose as many as were applicable. Eating less food or modifying dietary patterns was divided into two items (skipping meals, and eating less food without skipping meals) in the 2010-2011 survey: responses were combined in the final analysis to maintain consistency with the data of the 2008-2009 survey.

\section{3) Sociodemographic factors}

Height and weight were measured while subjects wore light clothing and no shoes, using a SECA225 apparatus (SECA Deutschland, Hamburg, Germany) and a GL-6000-20 apparatus (CASKOREA, Seoul, Korea), respectively. Body mass index (BMI) was calculated by dividing weight $(\mathrm{kg})$ by the square of height (m). Weight status was classified into underweight (BMI $\left.\leq 18.4 \mathrm{~kg} / \mathrm{m}^{2}\right)$, normal weight (BMI 18.5-22.9 kg/m²), overweight (BMI 23.0-24.9 kg/m²), and obese (BMI $\geq 25.0 \mathrm{~kg} / \mathrm{m}^{2}$ ). 
Drinking status was classified into two groups (less than once per month, and one or more times per month) by frequency of alcohol intake. Marital status was categorized as married, unmarried, and other (divorced, separated, or widowed). Education level was categorized as college graduate or higher, high school graduate, and middle school graduate or less. The household equivalent income was split into tertiles (high, middle, and low) for each survey year. Occupation was classified as non-manual workers, manual workers, and other (students, homemakers, and unemployed) using the Korean Standard Classification of Occupations that was revised in 2007.

\section{Statistical Analyses}

Analyses were performed after accounting for stratification, cluster, and sample weight based on the guidelines for use of the KNHANES' raw data. Data were expressed as mean \pm standard error (SE) or percentage \pm SE. The simple relationship between cotinine-verified smoking and each weight control method was assessed using the chi-square test. Logistic regression analyses were used to obtain the odds ratio (OR) and $95 \%$ confidence intervals (CI) of cotinine-verified smoking on each weight control method. All statistical analyses were performed using PASW SPSS ver. 18.0 (SPSS Inc., Chicago, IL, USA). A P-value $<0.05$ was considered statistically significant.

\section{RESULTS}

The study subjects were Korean women aged $\geq 19$ years who underwent assays of urinary cotinine and responded in the KNHANES, 2008-2011 that they had attempted weight control during the past 1 year. The smoking rate based on measurement of urinary cotinine was $12.4 \% \pm 0.8 \%$. Cotinine-verified smokers were significantly younger than cotinine-verified non-smokers ( $37.8 \pm 1.0$ years vs. $44.9 \pm 0.4$ years, $\mathrm{P}<0.001)$. The sociodemographic characteristics of smokers and non-smokers determined by urinary cotinine level are presented in Table 1 . Of the cotinine-verified smokers, $56.0 \% \pm 3.1 \%$ were assessed as non-smokers or ex-smokers using self-report questionnaires. In comparison, $0.3 \% \pm 0.1 \%$ of cotinine-verified non-smokers answered that they were current smokers. 
Table 1. General characteristics of the study subjects

\begin{tabular}{|c|c|c|c|}
\hline \multirow{2}{*}{ Characteristic } & \multicolumn{2}{|c|}{ Cotinine-verified smoking status } & \multirow{2}{*}{ P-value } \\
\hline & Non-smokers (unweighted $\mathrm{n}=3,678$ ) & Smokers (unweighted $\mathrm{n}=511$ ) & \\
\hline Age (y) & & & $<0.001$ \\
\hline$\leq 39$ & $36.7(1.1)$ & $60.5(3.3)$ & \\
\hline $40-59$ & $46.1(1.2)$ & $33.7(3.0)$ & \\
\hline$\geq 60$ & $17.2(1.1)$ & $5.8(2.0)$ & \\
\hline Weight status & & & 0.127 \\
\hline Underweight & $1.8(0.3)$ & $4.1(1.3)$ & \\
\hline Normal weight & $40.3(1.3)$ & $42.2(3.0)$ & \\
\hline Overweight & $23.5(1.1)$ & $21.4(3.0)$ & \\
\hline Obese & $34.4(1.3)$ & $32.3(2.9)$ & \\
\hline Self-reported smoking status & & & $<0.001$ \\
\hline Non-smokers & $95.6(0.5)$ & $48.8(3.2)$ & \\
\hline Ex-smokers & $4.1(0.5)$ & $7.2(1.9)$ & \\
\hline Current smokers & $0.3(0.1)$ & $44.0(3.1)$ & \\
\hline Alcohol intake (times/mo) & & & $<0.001$ \\
\hline$<1$ & $57.0(1.3)$ & $37.6(3.1)$ & \\
\hline$\geq 1$ & $43.0(1.3)$ & $62.4(3.1)$ & \\
\hline Marital status & & & $<0.001$ \\
\hline Married & $70.3(1.2)$ & $56.2(3.0)$ & \\
\hline Unmarried & $18.5(1.0)$ & $28.6(2.7)$ & \\
\hline Other $^{\dagger}$ & $11.2(0.9)$ & $15.3(2.6)$ & \\
\hline Education level & & & 0.002 \\
\hline$\leq$ Middle school & $32.3(1.3)$ & $22.1(3.1)$ & \\
\hline High school & $40.0(1.3)$ & $51.5(3.2)$ & \\
\hline$\geq$ College & $27.7(1.1)$ & $26.4(2.6)$ & \\
\hline Household income & & & 0.702 \\
\hline Low & $22.1(1.3)$ & $24.3(3.1)$ & \\
\hline Middle & $36.7(1.3)$ & $34.4(3.0)$ & \\
\hline High & $41.2(1.4)$ & $41.3(3.2)$ & \\
\hline Occupation & & & 0.810 \\
\hline Non-manual & $19.5(0.9)$ & $19.9(2.3)$ & \\
\hline Manual & $30.7(1.2)$ & $32.4(3.1)$ & \\
\hline Other ${ }^{\ddagger}$ & $49.8(1.3)$ & $47.7(3.3)$ & \\
\hline
\end{tabular}

Values are presented as \% (standard error).

*By chi-square test. 'Including divorced, separated, and widowed. Including students, homemakers, and unemployed.

The frequency of use of each weight control method in cotinine-verified smokers and nonsmokers is shown in Table 2. Exercise, and eating less food or modifying dietary patterns, were the most commonly used methods for weight control in both groups. Compared to cotinine-verified nonsmokers, smokers more frequently chose fasting $(5.1 \% \pm 1.6 \%$ vs. $1.7 \% \pm 0.3 \%, \mathrm{P}=0.001)$, taking prescription diet-pills $(10.0 \% \pm 1.6 \%$ vs. $3.3 \% \pm 0.4 \%, \mathrm{P}<0.001)$, and taking nonprescription diet-pills $(5.0 \% \pm 1.3 \%$ vs. $1.4 \% \pm 0.3 \%, \mathrm{P}<0.001)$, and chose exercise less often $(62.8 \% \pm 3.2 \%$ vs. $69.6 \% \pm$ $1.2 \%, \mathrm{P}=0.042)$. 
Table 2. Frequency of use of each weight control method according to cotinine-verified smoking status

\begin{tabular}{|c|c|c|c|}
\hline \multirow{2}{*}{ Variable } & \multicolumn{2}{|c|}{ Cotinine-verified smoking status } & \multirow{2}{*}{ P-value } \\
\hline & Non-smokers (unweighted $\mathrm{n}=3,678$ ) & Smokers (unweighted $\mathrm{n}=511$ ) & \\
\hline Exercise & $69.6(1.2)$ & $62.8(3.2)$ & 0.042 \\
\hline $\begin{array}{l}\text { Eating less food or modifying dietary } \\
\text { patterns }\end{array}$ & $65.2(1.1)$ & $60.4(3.4)$ & 0.178 \\
\hline One-food diet & $1.6(0.3)$ & $3.5(1.3)$ & 0.063 \\
\hline Fasting & $1.7(0.3)$ & $5.1(1.6)$ & 0.001 \\
\hline Dietary supplements & $6.8(0.7)$ & $8.7(1.7)$ & 0.249 \\
\hline Korean herbal medicine & $3.5(0.5)$ & $3.8(1.1)$ & 0.767 \\
\hline Prescription diet-pills & $3.3(0.4)$ & $10.0(1.6)$ & $<0.001$ \\
\hline Nonprescription diet-pills & $1.4(0.3)$ & $5.0(1.3)$ & $<0.001$ \\
\hline
\end{tabular}

Values are presented as \% (standard error ).

*By chi-square test.

Adjusted ORs and 95\% CIs of cotinine-verified smoking on each weight control method were obtained using logistic regression analyses. Cotinine-verified smokers were more likely to attempt fasting (OR, 2.19; 95\% CI, 1.03 to 4.67), taking prescription diet-pills (OR, 2.37; 95\% CI, 1.47 to 3.82), and taking nonprescription diet-pills (OR, 3.46; 95\% CI, 1.71 to 6.98), and were less likely to attempt eating less food or modifying dietary patterns (OR, 0.71; 95\% CI, 0.51 to 0.99 ) than non-smokers (Table 3). Subgroup analyses were performed after stratifying subjects by age ( $\leq 39$ years and $\geq 40$ years) to assess whether the relationship between women's smoking and weight control methods varied with age. The relationship between cotinine-verified smoking and each weight control method did not differ depending on age. In both age groups, cotinine-verified smoking displayed a sustained and statistically significant relationship with a high likelihood of taking prescription diet-pills and nonprescription dietpills. The statistical significance of its relationship with a high likelihood of fasting and a low likelihood of eating less food or modifying dietary patterns declined with insufficient numbers of subjects within each subgroup (Table 4).

Table 3. Odds ratio of cotinine-verified smoking on each weight control method

\begin{tabular}{lccc}
\hline \multirow{2}{*}{\multicolumn{1}{c}{ Variable }} & \multicolumn{2}{c}{ Odds ratio (95\% confidence interval) } \\
\cline { 2 - 4 } & \multicolumn{1}{c}{${\text { Model } 1^{*}}^{*}$} & Model 2 & Model 3 $^{\dagger}$ \\
\hline Exercise & $0.78(0.58-1.04)$ & $0.81(0.60-1.09)$ & $0.87(0.63-1.19)$ \\
Eating less food or modifying dietary patterns & $0.70(0.52-0.95)^{\S}$ & $0.71(0.51-0.98)^{\S}$ & $0.71(0.51-0.99)^{\S}$ \\
One-food diet & $1.77(0.75-4.16)$ & $1.65(0.67-4.02)$ & $1.44(0.62-3.37)$ \\
Fasting & $2.48(1.23-5.02)^{\S}$ & $2.38(1.17-4.86)^{\S}$ & $2.19(1.03-4.67)^{\S}$ \\
Dietary supplements & $1.29(0.82-2.04)$ & $1.21(0.75-1.95)$ & $1.24(0.77-1.99)$ \\
Korean herbal medicine & $0.94(0.49-1.79)$ & $0.81(0.43-1.54)$ & $0.82(0.43-1.55)$ \\
Prescription diet pills & $2.54(1.62-3.98)^{\S}$ & $2.31(1.45-3.68)^{\S}$ & $2.37(1.47-3.82)^{\S}$ \\
Non-prescription diet pills & $3.72(1.85-7.49)^{\S}$ & $3.34(1.69-6.59)^{\S}$ & $3.46(1.71-6.98)^{\S}$ \\
\hline
\end{tabular}

*Adjusted by age. ${ }^{\dagger}$ Adjusted by age, weight status, alcohol intake. ${ }^{\star}$ Adjusted by age, weight status, alcohol intake, marital status, education level, household income, occupation. ${ }^{\S} \mathrm{P}$-value $<0.05$ by logistic regression analysis. 
Table 4. Odds ratio of cotinine-verified smoking on each weight control method after stratifying by age

\begin{tabular}{lcc}
\hline \multirow{2}{*}{\multicolumn{1}{c}{ Variable }} & \multicolumn{2}{c}{ Age group (y) } \\
\cline { 2 - 3 } & $\leq 39$, OR $(95 \% \mathrm{CI})^{*}$ & $\geq 40$, OR $(95 \% \mathrm{CI})^{*}$ \\
\hline Exercise & $0.73(0.50-1.08)$ & $0.99(0.60-1.63)$ \\
Eating less food or modifying dietary patterns & $0.78(0.52-1.17)$ & $0.69(0.41-1.16)$ \\
One-food diet & $1.03(0.40-2.69)$ & $1.97(0.69-5.57)$ \\
Fasting & $2.03(0.91-4.54)$ & $1.83(0.44-7.69)$ \\
Dietary supplements & $1.38(0.73-2.60)$ & $0.99(0.45-2.19)$ \\
Korean herbal medicine & $0.88(0.41-1.90)$ & $0.72(0.25-2.09)$ \\
Prescription diet-pills & $2.19(1.25-3.85)^{\dagger}$ & $3.21(1.31-7.83)^{\dagger}$ \\
Nonprescription diet-pills & $3.10(1.35-7.11)^{\dagger}$ & $5.21(1.96-3.85)^{\dagger}$ \\
\hline
\end{tabular}

OR: odds ratio, $\mathrm{CI}$ : confidence interval.

*Adjusted by weight status, alcohol intake, marital status, education level, household income, occupation. ${ }^{\dagger} \mathrm{P}$-value $<0.05$ by logistic regression analysis.

Further analyses sought to ascertain the relationship between women's smoking and each weight control method when smoking status was assessed using self-report questionnaires instead of assays of urinary cotinine. Although the assessment of smoking status was based on self-report, the results were almost consistent with those of the main analyses based on urinary cotinine level, except for a minimal alteration in ORs on exercise, eating less food or modifying dietary patterns, and fasting (Table 5).

Table 5. Odds ratio of self-reported smoking on each weight control method

\begin{tabular}{llll}
\hline \multirow{2}{*}{ Variable } & \multicolumn{2}{c}{ Odds ratio $(95 \%$ confidence interval) } \\
\cline { 2 - 4 } & \multicolumn{1}{c}{ Model 1 ${ }^{*}$} & Model 2 & Model 3 $^{\ddagger}$ \\
\hline Exercise & $0.55(0.37-0.82)^{\S}$ & $0.54(0.36-0.81)^{\S}$ & $0.57(0.37-0.87)^{\S}$ \\
Eating less food or modifying dietary patterns & $0.67(0.43-1.03)$ & $0.69(0.44-1.07)$ & $0.68(0.43-1.07)$ \\
One-food diet & $1.13(0.47-2.73)$ & $1.05(0.42-2.61)$ & $0.66(0.25-1.77)$ \\
Fasting & $3.06(1.63-5.74)^{\S}$ & $2.66(1.39-5.07)^{\S}$ & $2.13(0.97-4.66)$ \\
Dietary supplements & $1.00(0.54-1.84)$ & $0.92(0.49-1.72)$ & $0.99(0.51-1.91)$ \\
Korean herbal medicine & $0.93(0.39-2.19)$ & $0.83(0.35-1.97)$ & $1.06(0.44-2.57)$ \\
Prescription diet-pills & $2.98(1.79-4.97)^{\S}$ & $2.76(1.59-4.80)^{\S}$ & $2.85(1.60-5.06)^{\S}$ \\
Nonprescription diet-pills & $3.66(1.52-8.82)^{\S}$ & $3.30(1.42-7.68)^{\S}$ & $3.58(1.50-8.52)^{\S}$ \\
\hline
\end{tabular}

*Adjusted by age. ${ }^{\dagger}$ Adjusted by age, weight status, alcohol intake. ${ }^{\star}$ Adjusted by age, weight status, alcohol intake, marital status, education level, household income, occupation. ${ }^{\S} \mathrm{P}$-value $<0.05$ by logistic regression analysis.

\section{DISCUSSION}

This study aimed to investigate weight control methods related to cotinine-verified smoking among Korean women aged $\geq 19$ years who had attempted weight control during the past 1 year using data from the KNHANES, 2008-2011. Korean women smokers were more likely to attempt fasting and taking diet-pills to control their weight in an effort to achieve rapid weight reduction. These weight 
control behaviors can be fraught with harmful side effects. Yet, the women were less likely to choose dietary modification and exercise, which requires a constant effort over a prolonged time.

Unlike previous studies that relied on only self-report, we assessed smoking status objectively by measuring urinary cotinine. Some women smokers tend to describe themselves as non-smokers when their smoking status is only assessed by self-report in Korean society, reflecting a sociocultural background in which women's smoking is stigmatized. ${ }^{3)}$ Jung-Choi et al. ${ }^{2)}$ reported that among cotinine-verified smokers, $12.1 \%$ of men and $58.9 \%$ of women self-reported as non-smokers. Therefore, assessing the smoking status of Korean women using only self-report could result in measurement bias. Cotinine is a metabolite of nicotine with a long half-life, which is considered a reliable biochemical marker of current nicotine consumption. Measurement of urinary cotinine level is most preferred, because urine can be collected easily and non-invasively. ${ }^{11-13)}$ Since some of the KNHANES' participants underwent assays of urinary cotinine, we think that the KNHANES is a useful data source for the study of health and risk behaviors in Korean women smokers.

Most previous studies on the relationship between smoking and weight control behaviors have involved adolescents. In one study, current cigarette use was approximately twice as high among US high school students who engaged in unhealthy weight control behaviors including fasting, taking diet pills, powders, or liquids, and vomiting or taking laxatives, compared with those who did not. ${ }^{14)}$ Problem behaviors, such as smoking, alcohol consumption, and drug use tend to cluster during adolescence. Unhealthy weight control behaviors may also be part of the problem behavior cluster in adolescents. ${ }^{5}$ In the present study, smoking in Korean adult women was related to unhealthy weight control behaviors, including fasting and taking diet-pills regardless of age, although there have been few previous studies on the relationship between smoking and weight control behaviors among adult women. This result, which is similar to that of most previous studies on adolescents living in Western societies, may be associated with the negative perception of women's smoking in Korean society. Because women's smoking is stigmatized as much as adolescents' smoking in Korean society, ${ }^{15)}$ Korean adult women smokers may share some common characteristics with adolescent smokers.

There has also been societal pressure concerning a slimmer body shape for woman in Korean society. Among women aged $\geq 19$ years who participated in the Community Health Survey, Seoul, 2013 , more than $70 \%$ responded that they had tried to lose or maintain their body weight during the past 1 year. ${ }^{16)}$ Furthermore, a recent study based on data from the KNHANES, 2007-2010, showed that even among underweight women in whom weight control may do harm, approximately a quarter had attempted weight control during the past 1 year. ${ }^{17)}$ Some women use smoking as means of weight control $^{4)}$ and numbers of women smokers have concerns about weight gain after smoking cessation. ${ }^{18)}$ Moreover, Pomerleau et al. ${ }^{19)}$ demonstrated that women smokers with strong concerns about postcessation weight gain were more likely to engage in a variety of unhealthy weight control behaviors, including use of diet pills, diuretics, and vomiting. We think that concerns about post-cessation weight gain could explain the main result of our study that Korean women smokers are more likely to attempt extreme weight control methods with potentially harmful effects than non-smokers. 
This study has several limitations that should be carefully considered. Cotinine level was measured from only a single urine sample, and subjects using nicotine replacement therapy or electronic cigarettes could not be excluded. However, we think that other sources of nicotine may not affect the results of our study because the results were almost similar when women's smoking was assessed using self-report instead of urinary cotinine level. We could not infer causality or temporality because of the cross-sectional design of this study. In addition, recall bias might occur when the subjects reported on every method attempted to control weight during the past 1 year.

Despite these limitations, our study shows that Asian adult women's smoking was independently related to weight control methods with potentially harmful effects for the first time. The other strengths of our study include that data were from a nationally representative sample in Korea, and smoking status was assessed, not only using subjective responses on self-report questionnaires, but also the objective measurement of urinary cotinine.

Korean women are known to have a very low smoking rate, which results from under-reporting of smoking in self-reported surveys. The actual smoking rate among Korean women is higher than $10 \%$ and may continue to increase gradually. ${ }^{9}$ In addition, some Korean women use extreme weight control methods with potentially unhealthful effects, responding to the intense socio-cultural pressure to be thin. In the present study, Korean women smokers were more likely to attempt fasting and taking dietpills to control their weight. These weight control behaviors may harm their health. The women were less likely to choose weight control methods, such as dietary modification and exercise, which requires constant effort for a prolonged time. Concerns about post-cessation weight gain can be a major barrier for women smokers to quit smoking. We believe that instruction regarding healthy methods to control weight can help women smokers to stop smoking and to improve their health.

\section{CONFLICT OF INTEREST}

No potential conflict of interest relevant to this article was reported.

\section{REERENCES}

1. French DJ, Jang SN, Tait RJ, Anstey KJ. Cross-national gender differences in the socioeconomic factors associated with smoking in Australia, the United States of America and South Korea. Int J Public Health 2013;58:345-53.

2. Jung-Choi KH, Khang YH, Cho HJ. Hidden female smokers in Asia: a comparison of self-reported with cotinine-verified smoking prevalence rates in representative national data from an Asian population. Tob Control 2012;21:536-42.

3. Lee DR, Kim HS, Lee J. The Characteristics of false respondents on a self-reported smoking survey of Korean 
women: Korean National Health and Nutrition Examination Survey, 2008. Korean J Fam Med 2014;35:28-34.

4. Fidler JA, West R. Self-perceived smoking motives and their correlates in a general population sample. Nicotine Tob Res 2009;11:1182-8.

5. French SA, Jeffery RW. Weight concerns and smoking: a literature review. Ann Behav Med 1995;17:234-44.

6. Neumark-Sztainer D, Story M, Dixon LB, Murray DM. Adolescents engaging in unhealthy weight control behaviors: are they at risk for other health-compromising behaviors? Am J Public Health 1998;88:952-5.

7. Wee CC, Rigotti NA, Davis RB, Phillips RS. Relationship between smoking and weight control efforts among adults in the United States. Arch Intern Med 2001;161:546-50.

8. Hur YI, Cho YG, Kang JH, Park HA, Kim KW, Park NR, et al. Weight control methods related to depressive symptoms among adults living in Seoul. Korean J Fam Med 2011;32:21-8.

9. Korean Ministry of Health and Welfare, Korea Centers for Disease Control and Prevention. Korea health statistics 2011: Korea National Health and Nutrition Examination Survey (KNHANES V-2). Seoul: Korean Ministry of Health and Welfare; 2012.

10. SRNT Subcommittee on Biochemical Verification. Biochemical verification of tobacco use and cessation. Nicotine Tob Res 2002;4:149-59.

11. Lerman C, Orleans CT, Engstrom PF. Biological markers in smoking cessation treatment. Semin Oncol 1993;20:359-67.

12. Jarvis MJ, Tunstall-Pedoe H, Feyerabend C, Vesey C, Saloojee Y. Comparison of tests used to distinguish smokers from nonsmokers. Am J Public Health 1987;77:1435-8.

13. Kang YH, Lee YJ, Kim HK, Yun YH, Jeong SY, Lee JS, et al. Usefulness of urinary cotinine test to distinguish smokers from nonsmokers. Korean J Lab Med 2003;23:92-7.

14. Johnson JL, Eaton DK, Pederson LL, Lowry R. Associations of trying to lose weight, weight control behaviors, and current cigarette use among US high school students. J Sch Health 2009;79:355-60.

15. Cho HJ, Khang YH, Jun HJ, Kawachi I. Marital status and smoking in Korea: the influence of gender and age. Soc Sci Med 2008;66:609-19.

16. Korea Centers for Disease Control and Prevention. 2013 Community health survey report. Cheongju: Korea Centers for Disease Control and Prevention; 2014.

17. Choi OJ, Cho YG, Kang JH, Park HA, Kim KW, Hur YI, et al. Weight control attempts in underweight Korean adults: Korea National Health and Nutrition Examination Survey, 2007-2010. Korean J Fam Med 2013;34:393-402.

18. Levine MD, Perkins KA, Marcus MD. The characteristics of women smokers concerned about postcessation weight gain. Addict Behav 2001;26:749-56.

19. Pomerleau CS, Zucker AN, Stewart AJ. Characterizing concerns about post-cessation weight gain: results from a national survey of women smokers. Nicotine Tob Res 2001;3:51-60. 